\title{
SOME PROBABILITY LIMIT THEOREMS
}

\author{
BY FRANK SPITZER ${ }^{1}$
}

Communicated by Mark Kac, January 5, 1959

We are concerned with the partial sums $S_{0}=0, S_{n}=X_{1}+\cdots+X_{n}$ of identically distributed independent random variables $X_{i}$ with mean zero and finite positive variance, i.e.

$$
E\left(X_{i}\right)=0, \quad 0<\sigma^{2}\left(X_{i}\right)=\sigma^{2}<\infty .
$$

Some of the present results describe new phenomena, whereas others are refinements of known theorems. While they deal with the limiting behavior of certain functionals of the partial sums, they appear to be of a type which cannot be reduced to the study of a functional of the Wiener process. With the exception of Theorem 6, nothing but (1) will be assumed about the common distribution of the $X_{i}$.

We define the probabilities:

$$
\begin{aligned}
& c_{k}=\operatorname{Pr}\left[S_{k}>0\right], \quad k \geqq 1 ; \\
& p_{0}=1, \quad p_{n}=\operatorname{Pr}\left[S_{1}>0, \cdots, S_{n}>0\right], \quad n \geqq 1 ; \\
& q_{0}=1, \quad q_{n}=\operatorname{Pr}\left[S_{1} \leqq 0, \cdots, S_{n} \leqq 0\right], \quad n \geqq 1 ;
\end{aligned}
$$

and the random variables (which by virtue of (1) exist and are finite with probability 1$)$ :

$Z=$ the first positive term in the infinite sequence $S_{1}, S_{2}, \ldots$;

$N_{n}=$ the number of positive terms in the finite sequence $S_{1}$, $S_{2}, \cdots, S_{n}$

$N_{A}(I)=$ the number of terms $S_{k}$ in the infinite sequence $S_{1}, S_{2}, \cdots$, such that $S_{k} \in I$ and $S_{i} \leqq A$ for $i=1,2, \cdots, k$;

$N_{A}^{*}(I)=$ the number of terms $S_{k}$ in the infinite sequence $S_{1}, S_{2}, \cdots$, such that $S_{k} \in I$ and $\left|S_{i}\right| \leqq A$ for $i=1,2, \cdots, k$.

Here $A$ is a positive number and $I$ is a closed bounded interval. $\mu(I)$ will denote the length of $I$ when the $X_{i}$ are nonlattice random variables, and the number of integers in $I$ when the $X_{i}$ are lattice random variables such that the smallest group containing all possible values of all the partial sums $S_{n}$ is the group of all integers.

Theorem 1. The series $\sum_{1}^{\infty} k^{-1}\left(1 / 2-a_{k}\right)$ converges (conditionally; probably not always absolutely).

The constant $c=\exp \sum_{1}^{\infty} k^{-1}\left(1 / 2-a_{k}\right)$ plays an important role in

\footnotetext{
${ }^{1}$ Research supported by the ONR at the University of Minnesota.
} 
what follows. Note that always $0<c<\infty$, while $c=1$ for symmetric random variables $X_{i}$ with a continuous distribution.

Theorem 2.

$$
E(Z)=c \cdot \sigma / 2^{1 / 2}
$$

THEOREM 3.

$$
\lim _{n \rightarrow \infty}(n \pi)^{1 / 2} p_{n}=\frac{1}{c}, \quad \lim _{n \rightarrow \infty}(n \pi)^{1 / 2} q_{n}=c .
$$

The methods of proof of these results are refinements of those in $[1 ; 4 ; 5]$. The moment generating function of $Z$ was first discovered by Baxter. In the simplest case, when the $X_{i}$ are symmetric with a continuous c.d.f. the proof of Theorem 2 follows from Karamata's Tauberian theorem applied to Baxter's identity

$$
\begin{gathered}
E\left[e^{-\lambda Z}\right]=1-\exp \left\{\frac{1}{2 \pi} \int_{-\infty}^{\infty} \frac{\lambda}{\lambda^{2}+u^{2}} \log [1-\phi(u)] d u\right\}, \\
\lambda>0, \quad \phi(u)=E\left[e^{i u X_{k}}\right] .
\end{gathered}
$$

THEOREM 4.

$$
(k(n-k))^{1 / 2} \operatorname{Pr}\left[N_{n}=k\right]=1 / \pi+o(1),
$$

where $o(1)$ is a function of $k$ and $n$ such that $o(1) \rightarrow 0$ as $\min (k, n-k)$ $\rightarrow \infty$.

This is an immediate corollary of Theorem 3 combined with Sparre Andersen's theorem [1]

$$
\operatorname{Pr}\left[N_{n}=k\right]=p_{k} q_{n-k} .
$$

It implies, but is stronger than, the celebrated arc-sine law for identically distributed random variables $[1 ; 2 ; 4]$.

THEOREM 5.

$$
\lim _{n \rightarrow \infty} E\left[\frac{S_{n}}{\left(n \sigma^{2}\right)^{1 / 2}} \mid S_{1} \geqq 0, S_{2} \geqq 0, \cdots, S_{n} \geqq 0\right]=\left(\frac{\pi}{2}\right)^{1 / 2} .
$$

This result follows easily from Theorems 2 and 3. It gains interest by comparison to

$$
\lim _{n \rightarrow \infty} E\left[\frac{S_{n}}{\left(n \sigma^{2}\right)^{1 / 2}} \mid S_{n} \geqq 0\right]=\left(\frac{2}{\pi}\right)^{1 / 2} .
$$


THEOREM 6. The $X_{i}$ are assumed to be symmetric a-periodic lattice with finite positive variance, i.e. random variables

$$
\begin{aligned}
c_{k}=\operatorname{Pr}\left[X_{i}=k\right]= & \operatorname{Pr}\left[X_{i}=-k\right]=c_{-k}, \quad 0<\sigma^{2}=\sum_{-\infty}^{\infty} k^{2} c_{k}<\infty, \\
& \text { g.c.d. }\left[k \mid c_{k}>0, k \geqq 1\right]=1 .
\end{aligned}
$$

The interval $I$ is assumed to be a single, fixed integer, so that $\mu(I)=1$. Then

$$
\begin{aligned}
& \lim _{A \rightarrow \infty} \operatorname{Pr}\left[N_{A}(I) \leqq \frac{2 A}{\sigma^{2}} \mu(I) x\right]= \begin{cases}1-e^{-x}, & x \geqq 0, \\
0, & x<0 .\end{cases} \\
& \lim _{A \rightarrow \infty} \operatorname{Pr}\left[N_{A}^{*}(I) \leqq \frac{A}{\sigma^{2}} \mu(I) x\right]= \begin{cases}1-e^{-x}, & x \geqq 0, \\
0 & x<0 .\end{cases}
\end{aligned}
$$

There is a close connection between this result and the considerations behind Theorem 2. Both are expressions of the asymptotic behavior of the resolvent of the substochastic transition operator of the stochastic process $\left\{S_{n}\right\}$ in the presence of an absorbing barrier (at $+A$ in Part (a) and at $\pm A$ in Part (b)). The proof of Part (b) was found in collaboration with C. Stone [7], using methods from the theory of Toeplitz forms [3], where geometric means such as the last term in Equation (2) play a crucial role.

The intuitive content of Theorem 6 as an occupation time theorem makes it extremely plausible that it holds for arbitrary $X_{i}$ satisfying (1), integer-valued and aperiodic if they are of the lattice type, with $I$ and $\mu(I)$ given in the definition of the occupation times $N_{A}(I)$ and $N_{A}^{*}(I)$.

The proofs of Theorems 1 through 5 will appear in [6] and that of Theorem 6(b) in [7].

\section{REFERENCES}

1. E. Sparre Andersen, On the fluctuations of sums of random variables II, Math. Scand. vol. 2 (1954) pp. 195-223.

2. P. Erdös and M. Kac, On the number of positive sums of independent random variables, Bull. Amer. Math. Soc. vol. 53 (1947) pp. 1011-1020.

3. U. Grenander and G. Szegö, Toeplitz forms and their applications, University of California Press, Berkeley, 1958.

4. F. Spitzer, A combinatorial lemma and its application to probability theory, Trans. Amer. Math. Soc. vol. 82 (1956) pp. 323-339.

5. - The Wiener-Hopf equation whose kernel is a probability density, Duke Math. J. vol. 24 (1957) pp. 327-344.

6. - A Tauberian theorem and its probability interpretation, to appear in Trans. Amer. Math. Soc.

7. F. Spitzer and C. Stone, A class of Toeplitz forms and their application to probability theory, to appear in Illinois J. Math.

University of Minnesota 